\title{
Hallazgos anatomopatologicos en un bovino infectado con tuberculosis en Vicosa, Brasil
}

\section{Anatomopathological findings in a bovine infected with tuberculosis in Viçosa, Brazil}

\author{
Bernardo Reyes $\mathrm{B}^{1}$ M.Sc; José Cardona Á ${ }^{1^{*}} \mathrm{Ph} . \mathrm{D}$; \\ Donicer Montes V² Ph.D; Marlene Vargas V³ Ph.D. \\ ${ }^{1}$ Universidad de Córdoba. Facultad de Medicina Veterinaria y Zootecnia, Grupo de \\ Investigación en Medicina de Grandes Animales (MEGA), Montería, Colombia. \\ ${ }^{2}$ Universidad de Sucre. Facultad de Ciencias Agropecuarias, \\ Departamento de Zootecnia. Sincelejo, Colombia. \\ ${ }^{3}$ Universidad Federal de Viçosa, Departamento de \\ Medicina Veterinaria, Viçosa, Brasil.
}

\section{KEYWORDS:}

bovine tuberculosis; cattle; necropsy.

\section{ABSTRACT}

A case of bovine tuberculosis was reported in a Holstein female of approximately $350 \mathrm{~kg}$ in weight, which was attended by the animal pathology service of the Faculty of Veterinary Medicine and Zootechnics of the University of Viçosa, Brasil. The owner reported progressive wasting, due to the poor state of health it was decided to sacrifice the animal and later moved to the area of pathology where the necropsy was performed. Hairy, pale mucous membranes and marked cachexia were evidenced in the external inspection of the corpse, the anatomopathological findings at necropsy revealed important injuries such as: nodular case areas in muscles in the intercostal region, pleura, lung, liver and prescapular lymph nodes. The definitive diagnosis of the corpse was bovine tuberculosis.

\section{PALABRAS CLAVE:}

Bovinos; tuberculosis bovina; necropsia.
INFORMACIÓN

Recibido: 7-02-2018;

Aceptado: $18-06-2018$

Publicado: 02-07-2018

Correspondencia autor:

cardonalvarez@hotmail.com

\section{RESUMEN}

Se reportó un caso de tuberculosis bovina en una hembra Holstein de aproximadamente $350 \mathrm{~kg}$ de peso, la cual fue necropsiada por el servicio de patología animal de la facultad de Medicina Veterinaria y Zootecnia de la Universidad de Viçosa, Brasil. El propietario reporta emaciación progresiva, debido al pésimo estado de salud se decidió sacrificar el animal y posteriormente se trasladó al área de patología donde fue realizada la necropsia. Se evidencio pelaje hirsuto, mucosas pálidas y marcada caquexia a la inspección externa del cadáver, los hallazgos anatomopatológicas en la necropsia revelaron importantes lesiones como: áreas caseificadas nodulares en músculos en la región intercostal, pleura, pulmón, hígado y ganglios linfáticos pre-escapulares. El diagnóstico definitivo del cadáver fue tuberculosis bovina. 
Reyes et al - Hallazgos anatomopatologicos en bovino infectado con tuberculosis en Brasil

\section{INTRODUCCION}

La tuberculosis bovina (TB), es una enfermedad infecciosa crónica de los rumiantes producida por la bacteria mycobacterium bovis., que puede también afectar a cerdos, equinos, perros, gatos, zorros, rumiantes silvestres, búfalos, llama, alpaca, cerdos silvestres, primates no humanos y el hombre, la principal vía de infección en el bovino es la vía aerógena y en menor medida la ingestión de material contaminado. (ESTRADA et al., 2004; GONZALES Y MATTAR, 2010; RIVERA y GIMENEZ, 2010).

Esta enfermedad afecta la salud y el bienestar animal, causando graves pérdidas económicas para el sector ganadero en todo el mundo a través de sacrificio involuntario, restricciones de movimiento de animales, y el costo de los programas de control y erradicación (BANOS et al., 2017).

Los animales afectados con esta enfermedad son generalmente asintomáticos o pueden presentar síntomas característicos como emaciación progresiva, tos, disnea, aumento de los ganglios linfáticos y disminución en la producción. (OLIVEIRA et al., 20017).

Luego de la exposición al aerosol y la fagocitosis, Los macrófagos infectados ingresan al sistema linfático y son transportados hasta los ganglios linfáticos, Esta inmersión con macrófagos a su vez activará otros macrófagos y atraerá células T auxiliares al zona, Las células T activadas luego proceden a matar macrófagos infectados con micobacterias que conducen a la destrucción del tejido circundante, que en combinación con los macrófagos muertos o moribundos crea necrosis caseosa que forma un granuloma o lesión (O'HAGAN et al., 2015).

En los hallazgos post-mortem se reportan lesiones nodulares caseosas localizadas en el tejido pulmonar, linfonodo broncomediastinal, pleura, hígado y ganglios linfáticos mesentéricos así como lesiones caseosas-escleróticas-calcificadas en los ganglios retrofaringeo y peribronqueal. (ZANARDI et al, 2013)
El diagnóstico de la tuberculosis bovina se puede lograr mediante el uso de métodos de laboratorio convencionales como cultivo y frotis al microscopio, Estos métodos son económicos pero su sensibilidad es baja, recientemente, técnicas moleculares de mejor rendimiento y más asequibles han sido introducidas como PCR, el método de diagnóstico más utilizado para la detección de animales tuberculosos es la prueba de tuberculina, que puede realizarse de tres formas: por la prueba simple cervical, cervical comparativa o del pliegue caudal, otras pruebas diagnósticas utilizadas son el ensayo de interferóngamma(IFN-Y) y pruebas ELISA para detección de anticuerpos. (BHEMBE et al., 2017; OLIVEIRA et al., 2007; CASAL et al., 2017)

El objetivo de este estudio fue describir y documentar las características anatomopatologicas de un bovino infectado con tuberculosis en Viçosa, MG, Brasil.

\section{MATERIALES Y METODOS}

Una hembra bovina Holstein de un peso aproximado de $350 \mathrm{~kg}$ fue necropsiada por el servicio de patología de la Facultad de Medicina Veterinaria y Zootecnia de la Universidad de Viçosa, Brasil.

Los datos anamnesicos, consistieron en emaciación progresiva crónica, por lo que fue sacrificada y posteriormente trasladada al área de patología donde fue realizada la necropsia.

Se evidencio pelaje hirsuto, mucosas pálidas y marcada caquexia a la inspección externa del cadáver, los hallazgos anatomopatologicos en la necropsia revelaron importantes lesiones como: áreas caseificadas nodulares en músculos en la región intercostal (Figura 1), pleura (Figura 2), pulmón (Figura 3), corazón (cuerdas tendinosas, válvulas y trabéculas cavernosas) (Figura 4), hígado (Figura 5) y ganglios linfáticos pre-escapulares, así como marcada cardiomegalia y secreciones mucopurulentas en las vías respiratorias (tráquea) (Figura 6). 


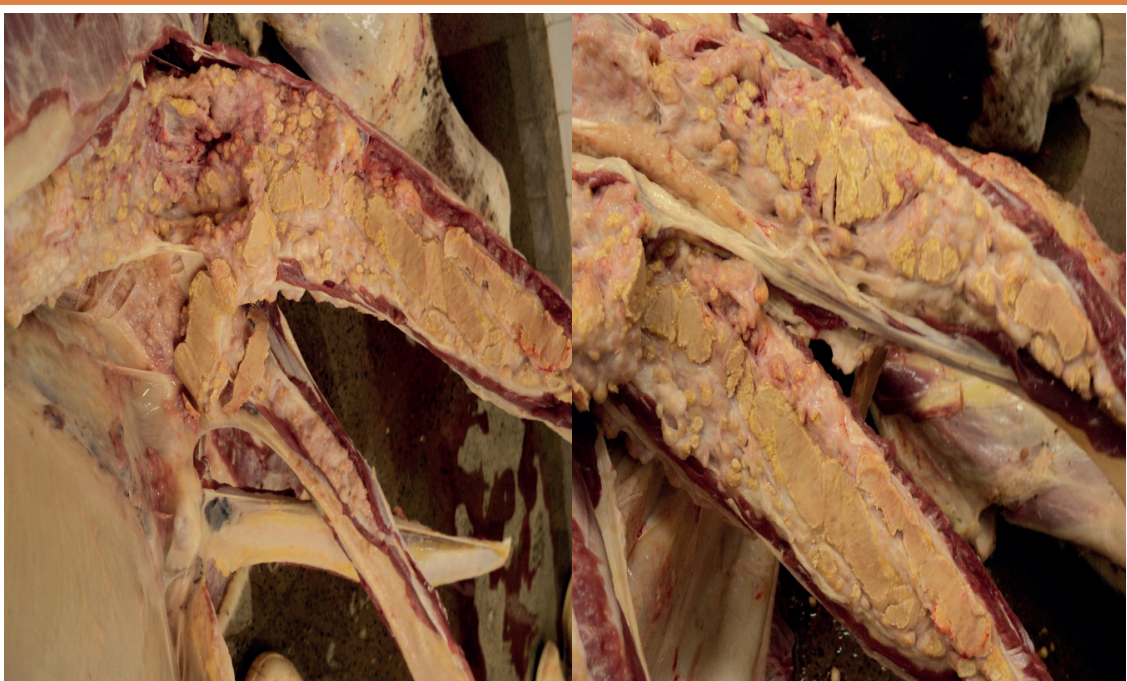

Figura 1. Lesiones nodulares caseificadas a nivel de la región intercostal.

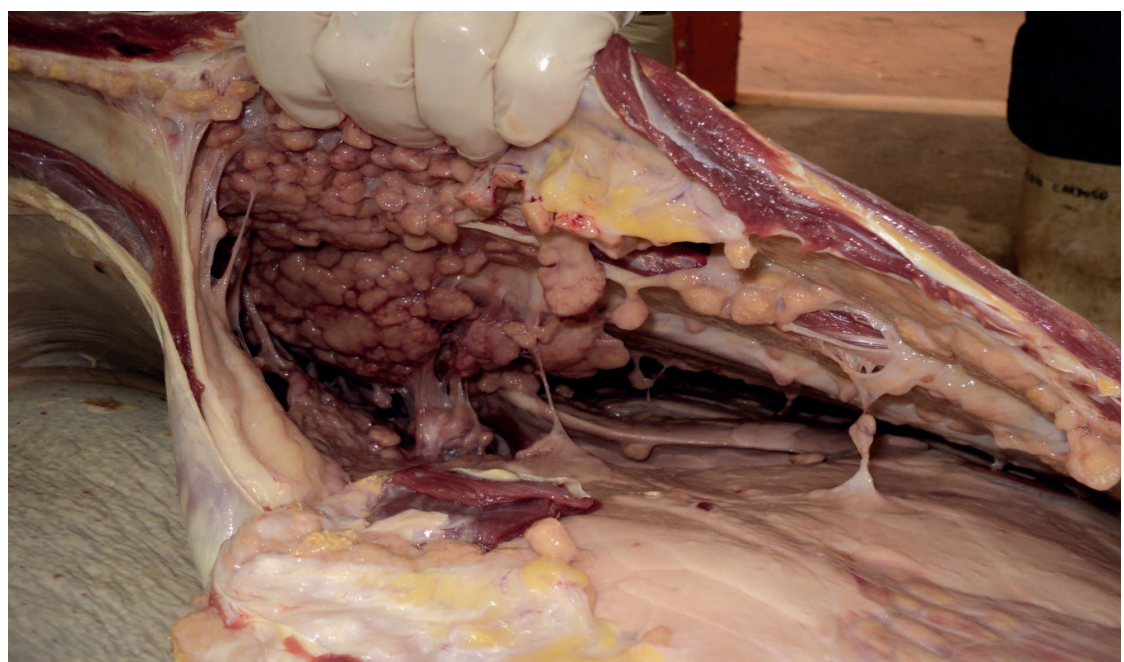

Figura 2. Nódulos caseificados localizados en la pleura parietal y visceral. 


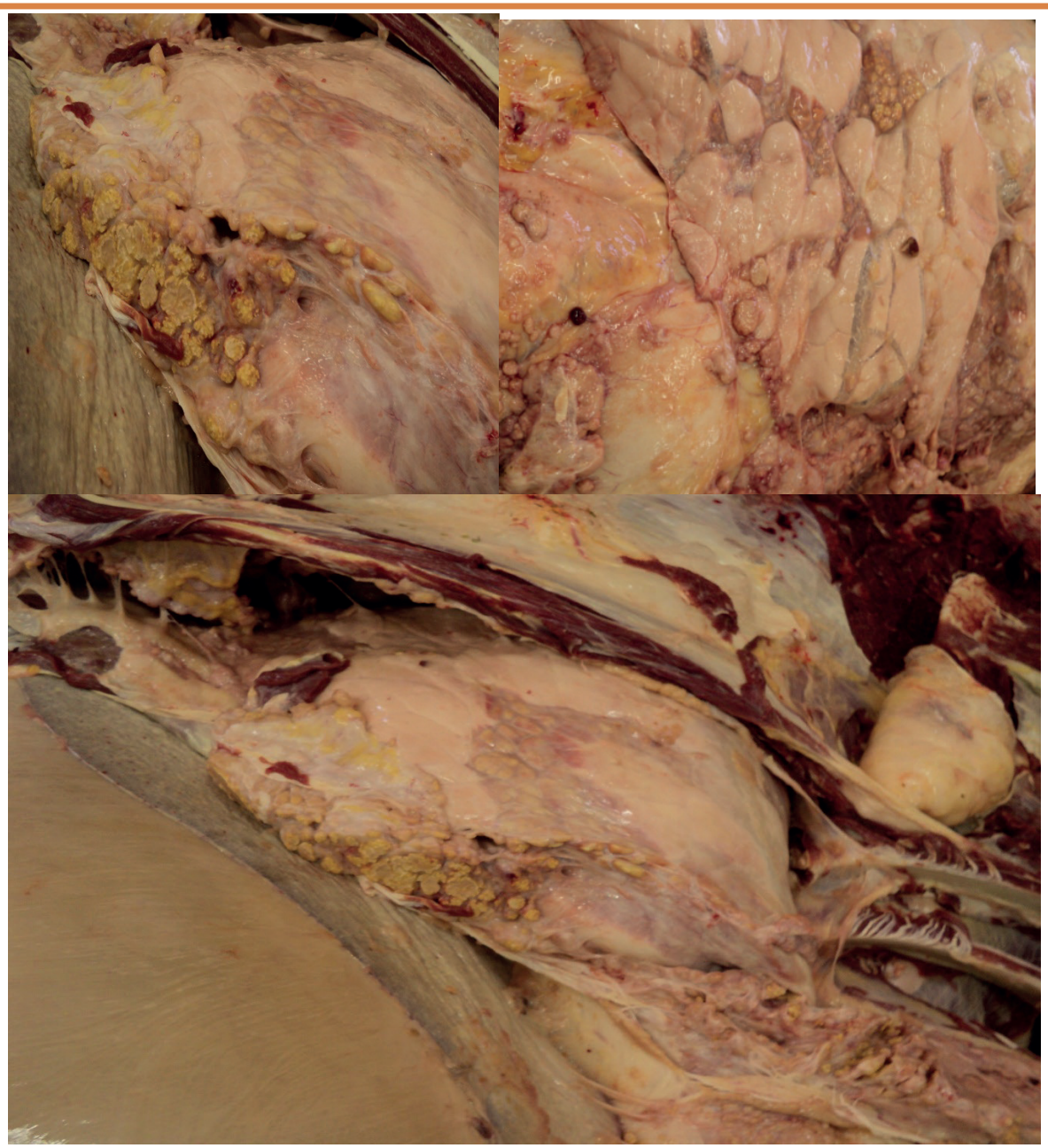

Figura 3. Lesiones nodulares caseificadas en pulmónes.

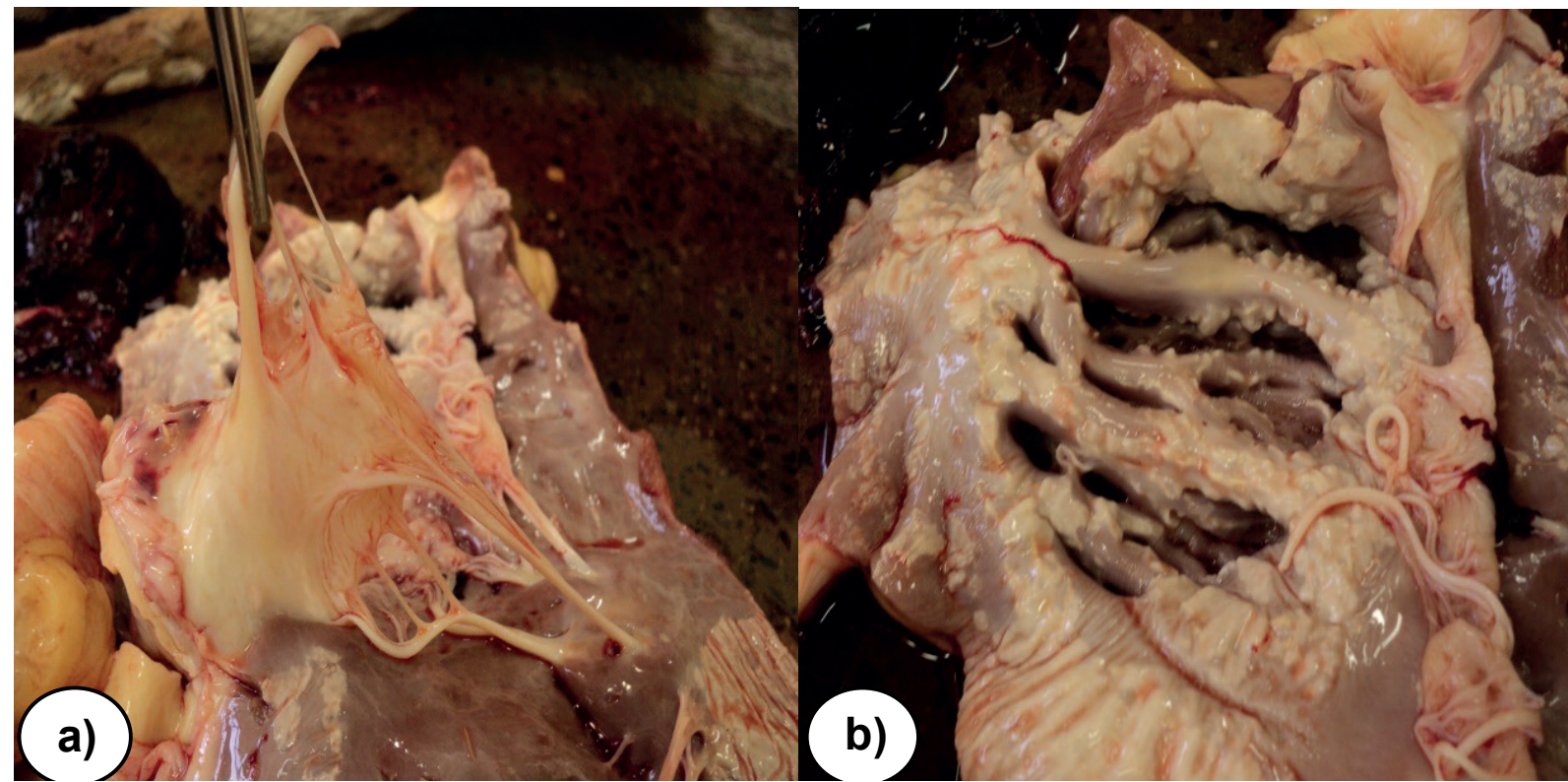

Figura 4. a) nódulos caseificados a nivel de válvulas y cuerdas tendinosas b) nódulos caseificados localizados en trabéculas cavernosas. 


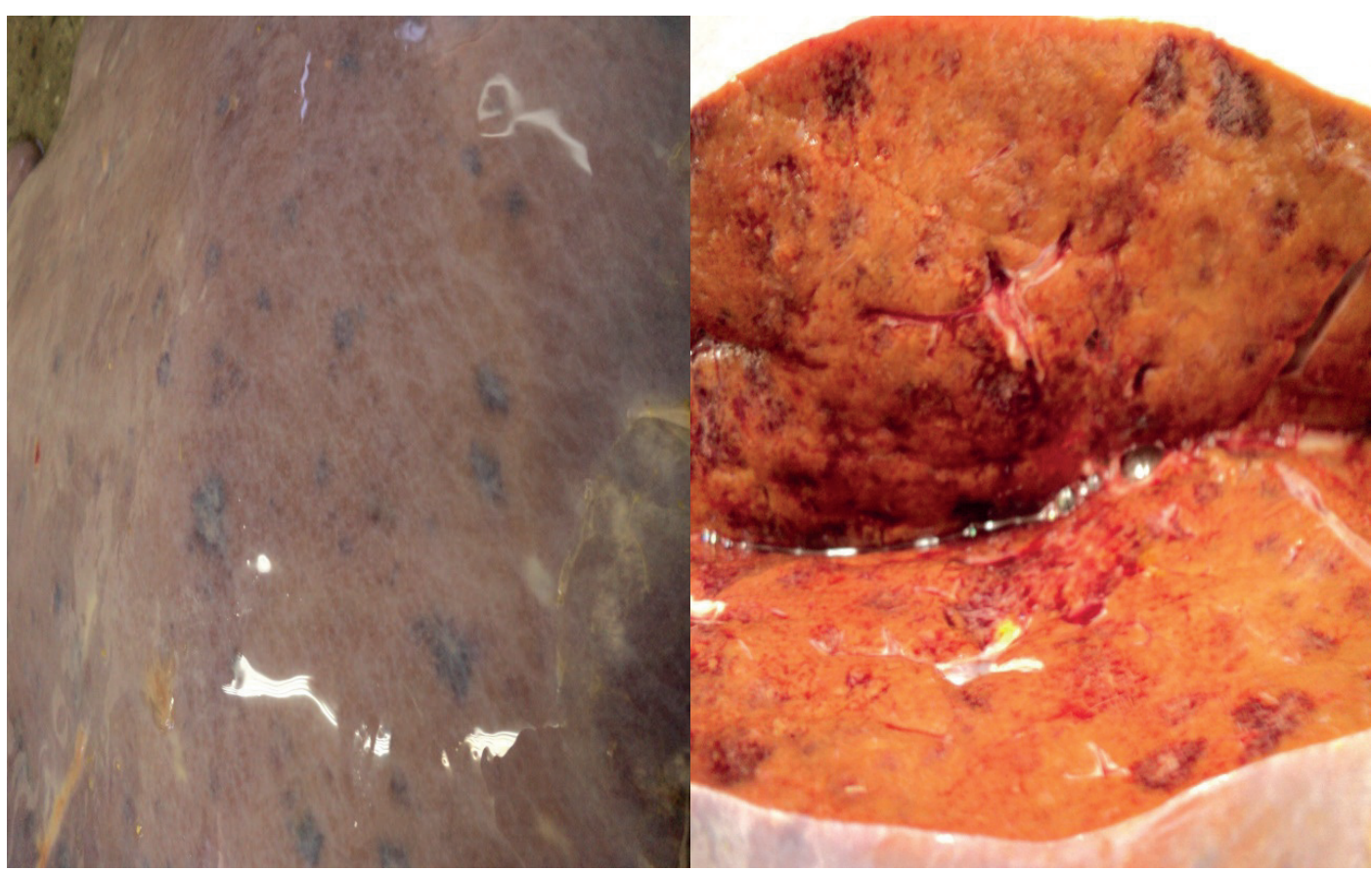

Figura 5. Lesiones nodulares caseificadas en el hígado.

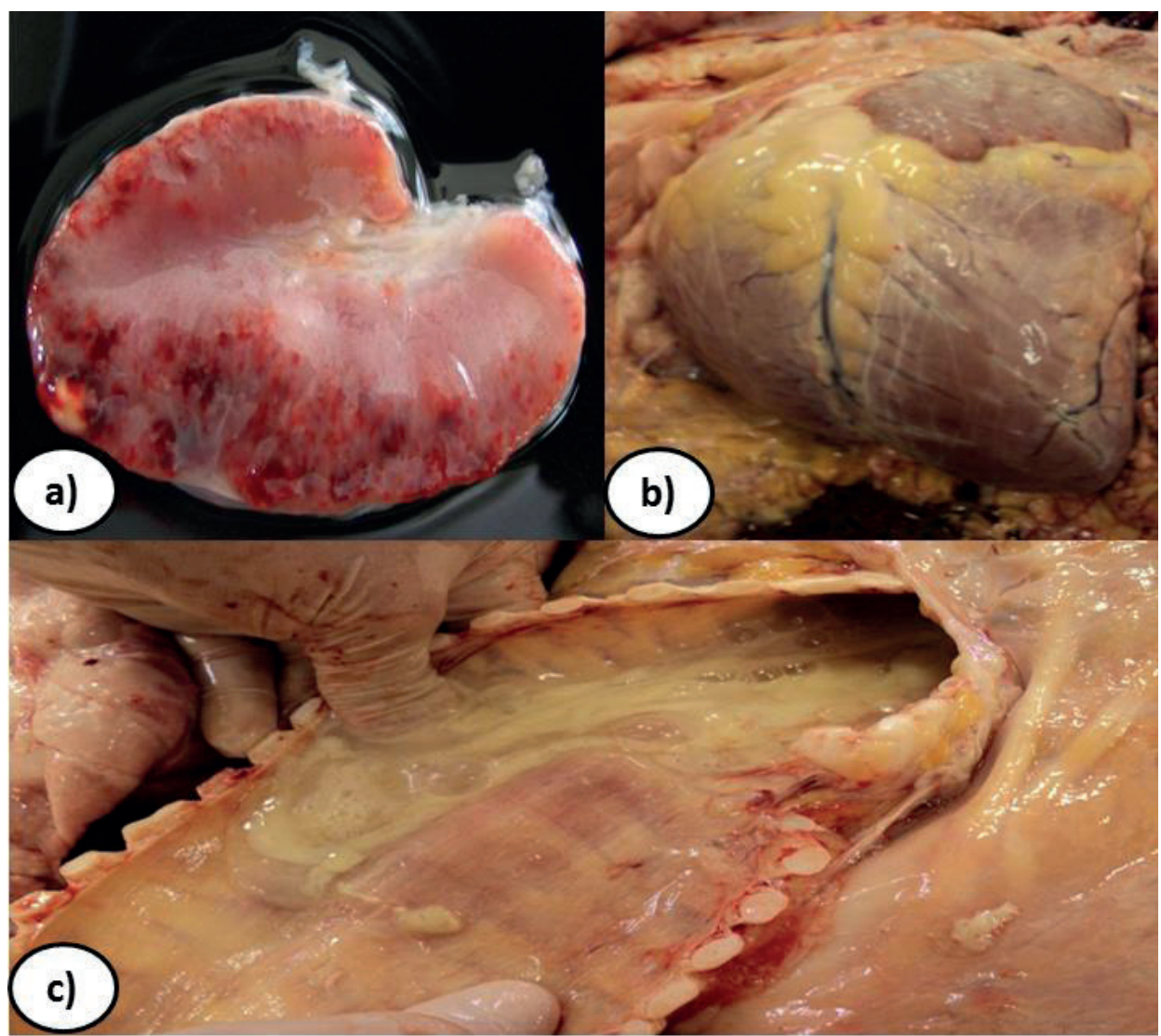

Figura 6. a). Lesiones nodurales caseificadas en los ganglios linfáticos preescapulares. b). Marcada cardiomegalia. c). Secreción mucopurulenta en la tráquea. 
Reyes et al - Hallazgos anatomopatologicos en bovino infectado con tuberculosis en Brasil

\section{RESULTADOS Y DISCUSIÓN}

Los hallazgos anatomopatologicos encontrados concuerdan con lo reportado por (ZANARDI et al, 2013) quien describió en el examen post-mortem de una vaca de 10 años de edad en la provincia de Bérgamo, Italia, hallazgos como lesiones nodulares caseosas localizadas en el tejido pulmonar, linfonodo broncomediastinal, pleura, hígado y ganglios linfáticos mesentéricos.

VORDERMEIER et al., (2012) reportan que estudios en etiopia datan porcentajes relativamente bajos de prevalencia de tuberculosis bovina tanto en pruebas de tuberculina como en datos de vigilancia de mataderos, en bovinos de razas cebuinas que fueron criados en sistemas de pastoreo, con un porcentaje de $0,8 \%$ a $10 \%$ mientras que en bovinos de sistemas lecheros intensivos, de razas exóticas la prevalencia es más alta (de $24 \%$ a 34\%) hecho que sugiere una relación entre raza y presentación de la tuberculosis bovina, pudiendo tener el ganado cebú algún tipo de resistencia a la infección en comparación con el ganado taurino, así, se podría atribuir la presentación de este caso a la raza del animal (mestizo), ya que posee $50 \%$ de sangre taurina.

BASYBEKOV et al., (2017) reportan que Los animales que reaccionaron a la tuberculina con hinchazón de la piel de $3 \mathrm{~mm}$ y más se consideran tuberculosos y fueron sacrificados, lo cual concuerda con el procedimiento realizado ante la sospecha de tuberculosis en este caso y de esta manera promover el control de esta enfermedad.

De acuerdo a las características anatomopatologicas, datos anamnesicos e inspección externa del cadáver, el diagnóstico definitivo del cadáver fue tuberculosis bovina.

\section{REFERENCIAS}

BANOS, G., WINTERS, M., MRODE, R., MITCHELL, A., BISHOP, S., WOOLLIAMS, J., COFFEY, M. 2017. Genetic evaluation for bovine tuberculosis resistance in dairy cattle. Journal of Dairy Science. 100(2):1272-1281.

BASYBEKOV, S., BAZARBAYEV, M., YESPEMBETOV, B., MUSSAEVA, A., KANATBAYEV, S., ROMASHEV, K., DOSSANOVA, A., YELEKEYEV, T., AKMATOVA, E., SYRYM, N. 2017. Diagnostics of tuberculosis and differentiation of nonspecific tuberculin reactions in animals. Brazilian journal of microbiology.

BHEMBE, N., JAJA, I., NWODO, U., OKOH, A., GREEN, E. 2017. Prevalence of tuberculous lymphadenitis in slaughtered cattle in Eastern Cape, South Africa. International Journal of Infectious Diseases. 61:27-37.

CASAL, C., INFANTES, J., RISALDE, M., DIEZ, A., DOMINGUEZ, M., MORENO, I., ROMERO, B., DE JUAN, L., SAEZ, J., JUSTE, R., GORTAZAR, C., DOMINGUEZ, L., BEZOS, J. 2017. Antibody detection tests improve the sensitivity of tuberculosis diagnosis in cattle. Research in Veterinary Science. 112:214-221.

ESTRADA, C., DIAZ, F., ARRIAGA, C., VILlegAS, N., PEREZ, R., GONZALEZ, D. 2004. Concordancia de la PCR y métodos rutinarios para el diagnóstico de tuberculosis bovina. Vet. Méx. 35 (3): 225-236.

GONZALES, M., MATTAR, S. 2010. ¿Ordeñando micobacterias del ganado? Impacto económico y en salud de Tuberculosis bovina y Paratuberculosis en Colombia?. Rev. MVZ. Cordoba. 15(2): 2037-2040. 
O'HAGAN, M., COURCIER, E., DREWE, J., GORDON, A., MC NAIE, J. 2015. Risk factors for visible lesions or positive laboratory tests in bovine tuberculosis reactor cattle in Northern Ireland. Preventive Veterinary Medicine. 120:283-290.

OLIVEIRA, I., MELO, H., HUDSON, CAMARA, A., DIAS, R., SOTO, B. 2007. Prevalência de tuberculose no rebanho bovino de Mossoró, Rio Grande do Norte. Braz. J. vet. Res. anim. Sci. 44(6):395-400.

RIVERA, S., GIMENE, F. 2010. La Tuberculosis Bovina en Venezuela: patogénesis, epidemiología, respuesta inmunitaria y nuevas alternativas para el diagnóstico. Redvet. 11(9):1-27.

VORDERMEIER, M., AMENI, G., BERG, S., BISHOP, S., ROBERTSON, B., ASEFFA, A., HEWINSON, R., YOUNG, D. 2012. The influence of cattle breed on susceptibility to bovine tuberculosis in Ethiopia. Comparative Immunology, Microbiology and Infectious Diseases. 35:227-232.

ZANARDI, G., BONIOTTI, B., GAFURRI, A., CASTRO, B., ZANONI, M., PACCIARINI, M. 2013. Tuberculosis transmission by Mycobacterium bovis in a mixed cattle and goat herd. Research in Veterinary Science. 95:430-433. 